\title{
Nonlinear dynamics in PT-symmetric lattices
}

\author{
Panayotis G. Kevrekidis ${ }^{1}$, Dmitry E. Pelinovsky ${ }^{2,3}$, and Dmitry Y.Tyugin ${ }^{3}$ \\ ${ }^{1}$ Department of of Mathematics and Statistics, University of Massachusetts, Amherst, MA 01003-9305, USA \\ ${ }^{2}$ Department of Mathematics and Statistics, McMaster University, Hamilton, Ontario, Canada, L8S 4 K1 \\ 3 Department of Applied Mathematics, Nizhny Novgorod State Technical University, Nizhny Novgorod, Russia
}

April 26, 2018

\begin{abstract}
We consider nonlinear dynamics in a finite parity-time-symmetric chain of the discrete nonlinear Schrödinger (dNLS) type. We work in the range of the gain and loss coefficient when the zero equilibrium state is neutrally stable. We prove that the solutions of the dNLS equation do not blow up in a finite time and the trajectories starting with small initial data remain bounded for all times. Nevertheless, for arbitrary values of the gain and loss parameter, there exist trajectories starting with large initial data that grow exponentially fast for larger times with a rate that is rigorously identified. Numerical computations illustrate these analytical results for dimers and quadrimers.
\end{abstract}

\section{Introduction}

Considerable recent interest in the physical literature have been devoted to lattices of the discrete nonlinear Schrödinger (dNLS) type with compensated gains and losses. For the onedimensional lattice, a prototypical, mono-parametric model takes the form

$$
i \frac{d u_{n}}{d t}=u_{n+1}-2 u_{n}+u_{n-1}+i \gamma(-1)^{n} u_{n}+\left|u_{n}\right|^{2} u_{n}
$$

where parameter $\gamma$ stands for the gain and loss coefficient. If the lattice is truncated at a finite chain, then the site index $n$ ranges from 1 to $2 N$ for a positive integer $N$ subject to the homogeneous Dirichlet boundary conditions $u_{0}=u_{2 N+1}=0$.

The dNLS model (11) represents one of the simplest discrete systems which are symmetric with respect to combined parity $(\mathrm{P})$ and time-reversal $(\mathrm{T})$ transformations. Hence, it is referred to as the PT-dNLS equation. Motivated by progress in optics both at the theoretical [8, 12, 17] and experimental [4, 18] levels, many researchers have studied nonlinear stationary states of few site configurations [3, 9, 10, 16, 19, 21, 22] as well as in infinite lattices [7, 20, 23].

In what follows, we will be particularly interested in dimers and quadrimers with alternating loss and gain in the adjacent sites. It should be noted that such configurations are directly amenable to the experimental considerations in [18]. In fact, in such finite waveguide arrays, due to the presence of iron doping, there unavoidably exists some loss in all the channels (lattice sites). However, the experimental procedure uses a mask covering some of the waveguides [6]. Then the ones that are not covered from the top are optically pumped and experience gain. 
There is considerable freedom in the selection of the mask so different configurations (such as loss, loss, gain, gain, or loss, gain, loss, gain) are feasible. However, the key feature is that because of the geometric characteristics of the waveguide (with each channel being about $4 \mu \mathrm{m}$ wide, but $30 \mathrm{~mm}$ long), only a homogeneous pump beam can be practically applied to all the gain channels, as in the case considered hereafter. Notice, however, that our analysis will be generalized beyond these configurations to arbitrary finite chains.

Our previous work [5] was devoted to the systematic analysis of nonlinear stationary states in the finite PT-symmetric chains. In particular, working in the range of the gain and loss coefficient $\gamma$ when the zero equilibrium state is neutrally stable [2, 15, we continued stationary states from the two limits characterized by small and large amplitudes of the stationary states. For these continuations, we modified the arguments of the Lyapunov-Schmidt reduction method to establish the existence of the solutions. We have also illustrated numerically that the stationary states are stable for small amplitudes and some of them are stable even for large amplitudes.

The nonlinear dynamics of oscillators with alternating loss and gain is an interesting problem in its own right. Aspects of such dynamics that have been previously explored include the following. Transmission resonances in a PT-symmetric dimer coupled with a linear dNLS chain were studied in [11. Asymmetric wave propagation through a finite PT-symmetric chain was observed in [1]. Nonlinear dynamics of wave packets near the phase transition point was addressed with the reduction to the nonlinear Klein-Gordon equation in [13, 14].

Our present paper studies the nonlinear dynamics of oscillators in the simplest finite PTsymmetric chains such as dimers and quadrimers. We shall prove the following three main results by using methods of the theory of differential equations. Although (most of) these results are expected on an intuitive level, the rigorous proof of these results appears to be a mathematical question of interest in its own right and can be seen, in some of the cases below, to present significant challenges. The three main results are summarized as follows:

R1 We prove that solutions of the PT-dNLS equation do not blow up in a finite time. This is achieved by a priori energy estimates for squared amplitudes of the nonlinear oscillators. In the Hamiltonian case $\gamma=0$, this result is equivalent to the conservation of the total sum of squared amplitudes, so the calculation proceeds in a fashion parallel to the corresponding conservation law and bears an extra step associated with Gronwall's lemma.

R2 For parameter values of the gain and loss coefficient $\gamma$ when the zero equilibrium state is neutrally stable (this regime is referred to as the exact PT-phase), we prove that the solutions of the finite PT-dNLS equation starting with small initial data remain bounded for all times. This is expected in Hamiltonian systems with the energy conservation when the zero equilibrium is stable. Nevertheless, the PT-dNLS equation with $\gamma \neq 0$ is non-Hamiltonian and lacks generally any conserved quantities.

R3 For the same values of $\gamma$, we prove that despite the fact that the zero equilibrium state is neutrally stable, there exist solutions of the finite PT-dNLS equation starting with sufficiently large initial data which grow exponentially fast for larger times. This result is perhaps the least expected among the three, because the values of $\gamma$ are inside the exact PT-phase, yet it can be anticipated since the balance between damped and gained 
oscillators is broken at large initial amplitudes, hence the amplitude of the oscillators with gain grows exponentially according to the linear law, in spite of the contributions of the nonlinear terms and interactions with other damped oscillators.

The article is structured as follows. Section 2 gives the rigorous proof of the three main results for the dimer. Although the dimer equations are fully integrable because of the presence of conserved quantities (reviewed in Appendix A), we develop qualitative methods of analysis, which become suitable for more complicated finite PT-symmetric chains. Section 3 outlines the proof of the three main results for the general case of finite PT-symmetric chains. Section 4 illustrates the main results with numerical computations of dimers and quadrimers. Section 5 contains a summarizing discussion and some consideration of future challenges.

Acknowledgments: The work of P.K. is partially supported by the US National Science Foundation under grants NSF-DMS-0806762, NSF-CMMI-1000337, and by the US AFOSR under grant FA9550-12-1-0332. The work of D.P. and D.T. is supported by the ministry of education and science of Russian Federation (Project 14.B37.21.0868).

\section{Nonlinear dynamics of a dimer}

Setting $a:=u_{1}$ and $b:=u_{2}$ for $N=1$, we consider the following system of two differential equations describing a PT-symmetric dimer:

$$
\left\{\begin{array}{l}
i \frac{d a}{d t}=b-i \gamma a+|a|^{2} a \\
i \frac{d b}{d t}=a+i \gamma b+|b|^{2} b
\end{array}\right.
$$

We fix the gain and loss parameter $\gamma$ in the interval $(0,1)$, which guarantees neutral stability of the zero equilibrium; this is the parametric interval of the exact PT-phase for this model. Indeed, for any $\gamma \in(0,1)$, the zero equilibrium point is neutrally stable with the eigenvalueeigenvector pairs:

$$
\lambda= \pm i \sqrt{1-\gamma^{2}}, \quad\left(\begin{array}{c}
a \\
b
\end{array}\right)=\left(\begin{array}{c}
1 \\
i \gamma \mp \sqrt{1-\gamma^{2}}
\end{array}\right) .
$$

The system of dimer equations (2) is fully integrable with two conserved quantities for any $\gamma \neq 0$. As a result, the three main results $\mathrm{R} 1-\mathrm{R} 3$ can be proven with the use of conserved quantities, as is outlined in Appendix A. Because the conserved quantities do not seem to exist for more complicated PT-symmetric configurations, such as quadrimers, we shall prove the same results using more general qualitative methods of the differential equation theory.

Proof of R1: It follows from the system of dimer equations (2) that

$$
\left\{\begin{array}{l}
\frac{d|a|^{2}}{d t}=-2 \gamma|a|^{2}+i(\bar{b} a-b \bar{a}), \\
\frac{d|b|^{2}}{d t}=2 \gamma|b|^{2}-i(\bar{b} a-b \bar{a}) .
\end{array}\right.
$$

Adding these two equations together, we obtain the balance equations for squared amplitudes

$$
\frac{d}{d t}\left(|a|^{2}+|b|^{2}\right)=2 \gamma\left(|b|^{2}-|a|^{2}\right) .
$$


By Gronwall' inequality, the balance equation (5) results in the a priori bound

$$
|a(t)|^{2}+|b(t)|^{2} \leq\left(\left|a_{0}\right|^{2}+\left|b_{0}\right|^{2}\right) e^{2 \gamma t}, \quad t \in \mathbb{R}
$$

where $a_{0}=a(0)$ and $b_{0}=b(0)$. A priori bound (6) proves that the amplitudes $|a(t)|$ and $|b(t)|$ do not blow up in a finite time.

Proof of R2: The key point of the proof is to rewrite the system of differential equations (44) in the integral form:

$$
|a(t)|^{2}=\left|a_{0}\right|^{2} e^{-2 \gamma t}+i \int_{0}^{t} e^{-2 \gamma(t-\tau)}[a(\tau) \bar{b}(\tau)-\bar{a}(\tau) b(\tau)] d \tau
$$

and

$$
|b(t)|^{2}=e^{2 \gamma t}\left(\left|b_{0}\right|^{2}-i \int_{0}^{t} e^{-2 \gamma \tau}[a(\tau) \bar{b}(\tau)-\bar{a}(\tau) b(\tau)] d \tau\right) .
$$

First, we show that the product $a b$ remains bounded as a function of $t$ for all times. Setting

$$
u:=\bar{a} b+a \bar{b}, \quad v:=i(a \bar{b}-\bar{a} b)
$$

and using the system of dimer equations (2), we obtain

$$
\left\{\begin{array}{l}
\frac{d u}{d t}=\left(|b|^{2}-|a|^{2}\right) v \\
\frac{d v}{d t}=\left(|b|^{2}-|a|^{2}\right)(2-u)
\end{array}\right.
$$

The system (9) reduces to the harmonic oscillator equation in the new temporal variable

$$
s(t):=\int_{0}^{t}\left(\left|b\left(t^{\prime}\right)\right|^{2}-\left|a\left(t^{\prime}\right)\right|^{2}\right) d t^{\prime} .
$$

Therefore, we obtain the exact solution of the system (9):

$$
\left\{\begin{array}{l}
u(t)=2+C_{1} \cos \left[\int_{0}^{t}\left(|b|^{2}-|a|^{2}\right) d t^{\prime}\right]+C_{2} \sin \left[\int_{0}^{t}\left(|b|^{2}-|a|^{2}\right) d t^{\prime}\right] \\
v(t)=-C_{1} \sin \left[\int_{0}^{t}\left(|b|^{2}-|a|^{2}\right) d t^{\prime}\right]+C_{2} \cos \left[\int_{0}^{t}\left(|b|^{2}-|a|^{2}\right) d t^{\prime}\right]
\end{array}\right.
$$

where $C_{1}$ and $C_{2}$ are arbitrary constants, which are uniquely defined by the initial conditions. From (10), we obtain

$$
|a(t) b(t)| \leq 1+\left|C_{1}\right|+\left|C_{2}\right|, \quad t \geq 0
$$

hence $a b$ is a bounded function of $t$ for all times. Note that this result also follows from the conservation law (40) in Appendix A.

Next, we show that the component $b(t)$ remains bounded for all times if and only if the solution of the system (2) satisfies the constraint

$$
\left|b_{0}\right|^{2}=i \int_{0}^{\infty} e^{-2 \gamma t}[a(t) \bar{b}(t)-\bar{a}(t) b(t)] d t .
$$

Indeed, since $|a b|$ is a bounded function of $t$, the integral in (8) is bounded for all $t \in \mathbb{R}_{+}$. Therefore, if the constraint (12) is not satisfied, it follows from the integral equation (8) that 
the solution $|b(t)|^{2}$ grows like $e^{2 \gamma t}$. On the other hand, if the constraint (12) is satisfied, the integral equation (8) takes the form

$$
|b(t)|^{2}=i e^{2 \gamma t} \int_{t}^{\infty} e^{-2 \gamma \tau}[a(\tau) \bar{b}(\tau)-\bar{a}(\tau) b(\tau)] d \tau,
$$

from which the global bound follows

$$
|b(t)|^{2} \leq \gamma^{-1} \sup _{t \in \mathbb{R}_{+}}|a(t) b(t)|, \quad t \in \mathbb{R}_{+}
$$

and implies that $\sup _{t \in \mathbb{R}_{+}}|b(t)|<\infty$. On the other hand, it follows from the integral equation (7) that

$$
|a(t)|^{2} \leq\left|a_{0}\right|^{2} e^{-2 \gamma t}+\gamma^{-1}\left(1-e^{-2 \gamma t}\right) \sup _{t \in \mathbb{R}_{+}}|a(t) b(t)|, \quad t \in \mathbb{R}_{+},
$$

hence $\sup _{t \in \mathbb{R}_{+}}|a(t)|<\infty$. Note that bound (14) implies

$$
\sup _{t \in \mathbb{R}_{+}}|b(t)| \leq \gamma^{-1} \sup _{t \in \mathbb{R}_{+}}|a(t)|
$$

It remains to show that the constraint (12) is satisfied for all solutions of the system of dimer equations (2) starting with small initial data $\left(a_{0}, b_{0}\right)$. Let $\delta:=\sqrt{\left|a_{0}\right|^{2}+\left|b_{0}\right|^{2}}$ be small. We would like to show that the amplitudes $|a|$ and $|b|$ do not grow significantly on the time scales of $t=\mathcal{O}\left(\delta^{-2}\right)$. This is achieved with the transformation of the system of dimer equations (2) to the normal coordinates near the zero equilibrium point.

Using the eigenvalues and eigenvectors in (3) near the zero equilibrium, we define the normal coordinates $c$ and $d$ by the transformation

$$
\left\{\begin{array}{l}
a=c-d\left(\sqrt{1-\gamma^{2}}+i \gamma\right) \\
b=c\left(\sqrt{1-\gamma^{2}}+i \gamma\right)+d
\end{array}\right.
$$

The system of dimer equations is now rewritten in coordinates $(c, d)$ :

$$
\left\{\begin{array}{l}
i \dot{c}=\sqrt{1-\gamma^{2}} c+\left(|c|^{2}+2|d|^{2}\right) c+d^{2} \bar{c}+2 i \gamma c^{2} \bar{d} \\
i \dot{d}=-\sqrt{1-\gamma^{2}} c+\left(2|c|^{2}+|d|^{2}\right) d+c^{2} \bar{d}-2 i \gamma d^{2} \bar{c}
\end{array}\right.
$$

Removing the linear terms by the phase rotation factors,

$$
c(t)=C(t) e^{-i \sqrt{1-\gamma^{2}} t}, \quad d(t)=d(t) e^{i \sqrt{1-\gamma^{2}} t},
$$

we obtain the cubic nonlinear system

$$
\left\{\begin{array}{l}
\dot{C}=-i\left(|C|^{2}+2|D|^{2}\right) C-i D^{2} \bar{C} e^{4 i \sqrt{1-\gamma^{2}} t}+2 \gamma C^{2} \bar{D} e^{-2 i \sqrt{1-\gamma^{2}} t} \\
\dot{D}=-i\left(2|C|^{2}+|D|^{2}\right) D-i C^{2} \bar{D} e^{-4 i \sqrt{1-\gamma^{2}} t}-2 \gamma D^{2} \bar{C} e^{2 i \sqrt{1-\gamma^{2}} t}
\end{array}\right.
$$

Since the solution exists globally, for any $T \in(0, \infty)$, let us define $\epsilon$ by

$$
\epsilon:=\sup _{t \in[0, T]} \sqrt{|C(t)|^{2}+|D(t)|^{2}}
$$


By Gronwall's inequality, we obtain from the system (19):

$$
|C(t)|^{2}+|D(t)|^{2} \leq\left(\left|C_{0}\right|^{2}+\left|D_{0}\right|^{2}\right) e^{(3+2 \gamma) \epsilon^{2} t}, \quad t \in[0, T] .
$$

Since $\left|C_{0}\right|^{2}+\left|D_{0}\right|^{2}=\mathcal{O}\left(\delta^{2}\right)$, we obtain $\epsilon=\mathcal{O}(\delta)$ if $T=\mathcal{O}\left(\epsilon^{-2}\right)$, that is, if $T=\mathcal{O}\left(\delta^{-2}\right)$.

Finally, if the constraint (12) is not satisfied, the solution $|b(t)|^{2}$ grows like $e^{2 \gamma t}$ and this growth on time $t=\mathcal{O}\left(\delta^{-2}\right)$ contradicts the bound (20), thanks to the transformations (16) and (18). Hence the constraint (12) is satisfied for all solutions of the dimer equations (2) starting with small initial data $\left(a_{0}, b_{0}\right)$, consequently these solutions remain bounded for all positive times.

Proof of R3: Let us consider the second equation of the system (44) rewritten as follows:

$$
\frac{d|b|^{2}}{d t}=2 \gamma|b|^{2}-i(\bar{b} a-b \bar{a})
$$

Let us choose the initial data $\left(a_{0}, b_{0}\right)$ to be sufficiently large so that

$$
2 \gamma\left|b_{0}\right|^{2}-i\left(\bar{b}_{0} a_{0}-b_{0} \bar{a}_{0}\right) \geq 2 \gamma\left|b_{0}\right|^{2}-2\left|a_{0}\right|\left|b_{0}\right| \geq 2 \gamma\left|b_{0}\right|^{2}-2\left(1+\left|C_{1}\right|+\left|C_{2}\right|\right)>0,
$$

where we have used the bound (11) on $\sup _{t \in \mathbb{R}_{+}}|a(t) b(t)|$. For instance, if $a_{0}=0$, then $C_{1}=-2$, $C_{2}=0$, and $b_{0}$ must satisfy the inequality $\left|b_{0}\right|^{2}>3 \gamma^{-1}$. Then, by the differential equation (21), $|b(t)|^{2}$ will grow and the inequality

$$
2 \gamma|b(t)|^{2}-i(\bar{b}(t) a(t)-b(t) \bar{a}(t)) \geq 2 \gamma|b(t)|^{2}-2\left(1+\left|C_{1}\right|+\left|C_{2}\right|\right)>0,
$$

will be preserved for all positive times. By the comparison principle for differential equations, $|b(t)|^{2}$ remains larger than the lower solution that grows exponentially like $e^{2 \gamma t}$. Then, by the integral equation (8), we conclude that the growth of $|b(t)|^{2}$ is exactly exponential like $e^{2 \gamma t}$.

Note that even if the component $b(t)$ for the gained oscillator grows exponentially, the component $a(t)$ for the damped oscillator remains bounded thanks to the bounds (11) and (15):

$$
\sup _{t \in \mathbb{R}_{+}}|a(t)|^{2} \leq\left|a_{0}\right|^{2}+\gamma^{-1} \sup _{t \in \mathbb{R}_{+}}|a(t) b(t)| \leq\left|a_{0}\right|^{2}+\gamma^{-1}\left(1+\left|C_{1}\right|+\left|C_{2}\right|\right),
$$

which is only defined by the initial data $\left(a_{0}, b_{0}\right)$. Moreover, $|a(t)|^{2}$ must decay exponentially as $e^{-2 \gamma t}$ to compensate the growth of $|b(t)|^{2}$ and to provide the uniform bound (11) for all positive times. Yet, while the growth of $|b(t)|^{2}$ is monotonic under these conditions, according to the above argument, the decay of $|a(t)|^{2}$ is typically oscillatory (see equation (49) in Appendix A).

It is also important to note that the above considerations provide a simple sufficient criterion for the exponential growth, namely

$$
\left|b_{0}\right|^{2}>\gamma^{-1}\left(1+\left|C_{1}\right|+\left|C_{2}\right|\right) .
$$

Given $a_{0}$ and $b_{0}$, coefficients $C_{1}$ and $C_{2}$ can be directly computed from the exact solution (10). Then, if the inequality (22) is satisfied, it can be immediately inferred that the amplitude $|b(t)|$ will grow indefinitely according to $e^{2 \gamma t}$ and $|a(t)|$ will correspondingly decay, so that their product remains bounded. 


\section{Nonlinear dynamics of a finite PT-symmetric chain}

We now consider the generalization of our results to a finite PT-symmetric chain, which is described by the PT-dNLS equation (11) for $n \in S_{N}:=\{1,2, \ldots, 2 N\}$, subject to the Dirichlet boundary conditions $u_{0}=u_{2 N+1}=0$. In the previous works [2, 5], it was proved that the zero equilibrium is neutrally stable for any $\gamma \in\left(-\gamma_{N}, \gamma_{N}\right)$, where

$$
\gamma_{N}:=2 \cos \left(\frac{\pi N}{1+2 N}\right) .
$$

When $N=1$, this corresponds to $\gamma_{1}=1$. In what follows, we fix $\gamma \in\left(0, \gamma_{N}\right)$. We are now ready to prove the three main results $\mathrm{R} 1-\mathrm{R} 3$ in the general case of finite $N \in \mathbb{N}$.

Proof of R1: For any $n \in S_{N}$, the squared amplitude satisfies the evolution equation

$$
\frac{d\left|u_{n}\right|^{2}}{d t}=2 \gamma(-1)^{n}\left|u_{n}\right|^{2}+g_{n}-g_{n-1}, \quad g_{n}:=i\left(u_{n} \bar{u}_{n+1}-\bar{u}_{n} u_{n+1}\right) .
$$

Adding up all equations, we obtain the balance equation for squared amplitudes

$$
\frac{d}{d t} \sum_{n \in S_{n}}\left|u_{n}\right|^{2}=2 \gamma \sum_{n \in S_{N}}(-1)^{n}\left|u_{n}\right|^{2}
$$

By Gronwall' inequality, the balance equation (24) results in the a priori bound

$$
\sum_{n \in S_{n}}\left|u_{n}(t)\right|^{2} \leq\left(\sum_{n \in S_{n}}\left|u_{n}(0)\right|^{2}\right) e^{2 \gamma t}, \quad t \in \mathbb{R} .
$$

Bound (25) proves that the set of amplitudes $\left\{\left|u_{n}(t)\right|\right\}_{n \in S_{N}}$ does not blow up in a finite time.

Proof of R2: We rewrite the differential equations (23) in the integral form, separately for odd $n$

$$
\left|u_{n}(t)\right|^{2}=\left|u_{n}(0)\right|^{2} e^{-2 \gamma t}+\int_{0}^{t} e^{-2 \gamma(t-\tau)}\left[g_{n}(\tau)-g_{n-1}(\tau)\right] d \tau
$$

and even $n$

$$
\left|u_{n}(t)\right|^{2}=e^{2 \gamma t}\left(\left|u_{n}(0)\right|^{2}+\int_{0}^{t} e^{-2 \gamma \tau}\left[g_{n}(\tau)-g_{n-1}(\tau)\right] d \tau\right) .
$$

We shall now prove that the set of components $\left\{g_{n}\right\}_{n \in S_{N}}$ remains bounded for all times. Setting

$$
f_{n}:=u_{n} \bar{u}_{n+1}+\bar{u}_{n} u_{n+1}, \quad g_{n}:=i\left(u_{n} \bar{u}_{n+1}-\bar{u}_{n} u_{n+1}\right)
$$

and using the PT-dNLS equation (10), we obtain

$$
\left\{\begin{array}{l}
\frac{d f_{n}}{d t}=\left(\left|u_{n+1}\right|^{2}-\left|u_{n}\right|^{2}\right) g_{n}+i\left(\bar{u}_{n-1} u_{n+1}-u_{n-1} \bar{u}_{n+1}\right)+i\left(u_{n} \bar{u}_{n+2}-\bar{u}_{n} u_{n+2}\right), \\
\frac{d g_{n}}{d t}=\left(\left|u_{n+1}\right|^{2}-\left|u_{n}\right|^{2}\right)\left(2-f_{n}\right)+\left(\bar{u}_{n-1} u_{n+1}+u_{n-1} \bar{u}_{n+1}\right)-\left(u_{n} \bar{u}_{n+2}+\bar{u}_{n} u_{n+2}\right) .
\end{array}\right.
$$

Using the variation of constants method, we write

$$
\left\{\begin{array}{l}
f_{n}(t)=2+C_{n}(t) e^{i s_{n}(t)}+D_{n}(t) e^{-i s_{n}(t)} \\
g_{n}(t)=i C_{n}(t) e^{i s_{n}(t)}-i D_{n}(t) e^{-i s_{n}(t)}
\end{array}\right.
$$


where

$$
s_{n}(t):=\int_{0}^{t}\left(\left|u_{n+1}\left(t^{\prime}\right)\right|^{2}-\left|u_{n}\left(t^{\prime}\right)\right|^{2}\right) d t^{\prime},
$$

and obtain the equivalent system of differential equations

$$
\left\{\begin{array}{l}
\frac{d C_{n}}{d t}=i\left(u_{n} \bar{u}_{n+2}-u_{n-1} \bar{u}_{n+1}\right) e^{-i s_{n}} \\
\frac{d D_{n}}{d t}=i\left(\bar{u}_{n-1} u_{n+1}-u_{n+2} \bar{u}_{n}\right) e^{i s_{n}}
\end{array}\right.
$$

Since $\bar{u}_{n} u_{n+1}=1+D_{n} e^{-i s_{n}}$, we integrate the second equation of the system (30) and obtain

$$
\bar{u}_{n} u_{n+1}=1+D_{n}(0) e^{-i s_{n}(t)}+i e^{-i s_{n}(t)} \int_{0}^{t}\left(\bar{u}_{n-1} u_{n+1}-u_{n+2} \bar{u}_{n}\right) e^{i s_{n}(\tau)} d \tau .
$$

If the amplitudes $\left|u_{n}\right|$ or $\left|u_{n+1}\right|$ are bounded for all times, then $\bar{u}_{n} u_{n+1}$ is bounded for all times from the Cauchy-Schwarz inequality $\left|g_{n}\right| \leq 2\left|u_{n}\right|\left|u_{n+1}\right|$. Therefore, to conclude that $g_{n}$ (or equivalently, $\left.\bar{u}_{n} u_{n+1}\right)$ remain bounded for all other solutions, it is sufficient to consider the case when either $\left|u_{n}\right|$ or $\left|u_{n+1}\right|$ or both grow as $t \rightarrow \infty$.

Assume that either $\left|u_{n}\right|$ or $\left|u_{n+1}\right|$ grow as $t \rightarrow \infty$, but not both. Without loss of generality, we assume that $\left|u_{n}\right|$ grows for even $n$. Then, for sufficiently large $t_{0}>0$, for which $\left|u_{n+1}\right|^{2}-\left|u_{n}\right|^{2}$ is sign-definite, we can write the integral term as follows:

$$
i \int_{t_{0}}^{t}\left(\bar{u}_{n-1} u_{n+1}-u_{n+2} \bar{u}_{n}\right) e^{i s_{n}(\tau)} d \tau=\int_{t_{0}}^{t} \frac{\bar{u}_{n-1} u_{n+1}-u_{n+2} \bar{u}_{n}}{\left|u_{n+1}\right|^{2}-\left|u_{n}\right|^{2}} \frac{d}{d \tau} e^{i s_{n}(\tau)} d \tau .
$$

Under the same assumption that $\left|u_{n}\right|$ grows for even $n$, it follows from the integral equation (27) that the amplitude $\left|u_{n}\right|$ grows as the precise exponential rate $e^{\gamma t}$. Similarly, $\left|u_{n+2}\right|$ may grow but is only allowed to grow at the same exponential rate. As a result, the integrand in (32) before the derivative term converges to a constant value as $t \rightarrow \infty$ exponentially fast. Therefore, integration by parts yields a uniform constant bound for all times including the limit $t \rightarrow \infty$. In this case, we conclude that there exist positive constants $\left\{G_{n}\right\}_{n \in S_{N}}$ such that

$$
\left|g_{n}(t)\right| \leq G_{n}, \quad t \geq 0, \quad n \in S_{N},
$$

hence $g_{n}$ is a bounded function of $t$ for all times.

It remains to exclude the case when both $\left|u_{n}\right|$ or $\left|u_{n+1}\right|$ grow simultaneously at the same rate such that $\left|u_{n+1}\right|^{2}-\left|u_{n}\right|^{2} \rightarrow 0$ as $t \rightarrow \infty$. Adding two amplitude balance equations (23) for odd $n$, we obtain

$$
\frac{d}{d t}\left(\left|u_{n+1}\right|^{2}+\left|u_{n}\right|^{2}\right)=2 \gamma\left(\left|u_{n+1}\right|^{2}-\left|u_{n}\right|^{2}\right)+g_{n+1}-g_{n-1} .
$$

If both $\left|u_{n}\right|$ or $\left|u_{n+1}\right|$ grow but $\left|u_{n+1}\right|^{2}-\left|u_{n}\right|^{2} \rightarrow 0$ as $t \rightarrow \infty$, then (34) implies that $\left|u_{n-1}\right|$ or $\left|u_{n+2}\right|$ grow at the same rate, in other words, all squared amplitudes $\left\{\left|u_{n}\right|^{2}\right\}_{n \in S_{N}}$ grow at the same rate and $\left|u_{n+1}\right|^{2}-\left|u_{n}\right|^{2} \rightarrow 0$ as $t \rightarrow \infty$ for all $n$. However, this clearly contradicts the balance equation (24). Therefore, this case is impossible.

By bound (33), functions $g_{n}$ are bounded for all $t$. It follows from the integral equation (26) that the squared amplitudes $\left|u_{n}(t)\right|^{2}$ are bounded for all odd $n$ (they correspond to the damped oscillators):

$$
\left|u_{n}(t)\right|^{2} \leq\left|u_{n}(0)\right|^{2} e^{-2 \gamma t}+(2 \gamma)^{-1}\left(1-e^{-2 \gamma t}\right) \sup _{t \in \mathbb{R}_{+}}\left(\left|g_{n}(t)\right|+\left|g_{n-1}(t)\right|\right), \quad t \in \mathbb{R}_{+},
$$


hence $\sup _{t \in \mathbb{R}_{+}}\left|u_{n}(t)\right|<\infty$ for odd $n$.

On the other hand, it follows from the integral equation (27) that $\left|u_{n}(t)\right|^{2}$ for all even $n$, corresponding to the gain oscillators, is bounded for all times if and only if the solution of the PT-dNLS equation (10) satisfies the constraint

$$
\left|u_{n}(0)\right|^{2}=\int_{0}^{\infty} e^{-2 \gamma t}\left[g_{n-1}(t)-g_{n}(t)\right] d t, \quad \text { for even } n \in S_{N} .
$$

If the constraint (36) is satisfied, the integral equation (26) takes the form

$$
\left|u_{n}(t)\right|^{2}=-e^{2 \gamma t} \int_{t}^{\infty} e^{-2 \gamma \tau}\left[g_{n}(\tau)-g_{n-1}(\tau)\right] d \tau,
$$

from which the global bound follows

$$
\left|u_{n}(t)\right|^{2} \leq(2 \gamma)^{-1} \sup _{t \in \mathbb{R}_{+}}\left(\left|g_{n}(t)\right|+\left|g_{n-1}(t)\right|\right), \quad t \in \mathbb{R}_{+}
$$

and implies that $\sup _{t \in \mathbb{R}_{+}}\left|u_{n}(t)\right|<\infty$ for even $n$. Using bound (35) and the inequality $\left|g_{n}\right| \leq$ $2\left|u_{n}\right|\left|u_{n+1}\right|$, we find from bound (38) that for all even $n$, we have

$$
\sup _{t \in \mathbb{R}_{+}}\left|u_{n}(t)\right| \leq \gamma^{-1} \sup _{t \in \mathbb{R}_{+}}\left(\left|u_{n-1}(t)\right|+\left|u_{n+1}(t)\right|\right) .
$$

The proof that the constraints (36) are satisfied for all solutions of the PT-dNLS equation (11) starting with small initial data is similar to the case of dimers. It is achieved with the transformation of the PT-dNLS equation to normal coordinates and subsequent control of the solution for long times that are inversely proportional to the squared size of the small initial data. This control is contradicted to the exponential growth of $\left|u_{n}(t)\right|^{2}$ like $e^{2 \gamma t}$ for even $n$ if the constraint (36) is not satisfied.

Proof of R3: Using the balance equation (23) for even $n$ and the global bound (33), we choose the initial data $\left\{u_{n}(0)\right\}_{n \in S_{N}}$ to be sufficiently large so that

$$
2 \gamma\left|u_{n}(0)\right|^{2}+g_{n}(0)-g_{n-1}(0) \geq 2 \gamma\left|u_{n}(0)\right|^{2}-G_{n}-G_{n-1}>0 .
$$

By the differential equation (23) for even $n$, the squared amplitude $\left|u_{n}(t)\right|^{2}$ will grow and the inequality

$$
2 \gamma\left|u_{n}(t)\right|^{2}+g_{n}(t)-g_{n-1}(t) \geq 2 \gamma\left|u_{n}(t)\right|^{2}-G_{n}-G_{n-1}>0,
$$

will be preserved for all positive times. By the comparison principle for differential equations, $\left|u_{n}(t)\right|^{2}$ for even $n$ remains larger than the lower solution that grows exponentially like $e^{2 \gamma t}$. Then, by the integral equation (26), we conclude that the growth of $\left|u_{n}(t)\right|^{2}$ for even $n$ is exactly exponential like $e^{2 \gamma t}$. Again, $\left|u_{n}(t)\right|^{2}$ for odd $n$ must decay exponentially as $e^{-2 \gamma t}$ to compensate the growth of $\left|u_{n}(t)\right|^{2}$ for even $n$ and to provide the uniform bound (33) for all positive times. As in the case of the dimer, the decay of the odd sites may be oscillatory, however, if the above inequality holds, the indefinite growth of the even sites is monotonic. 

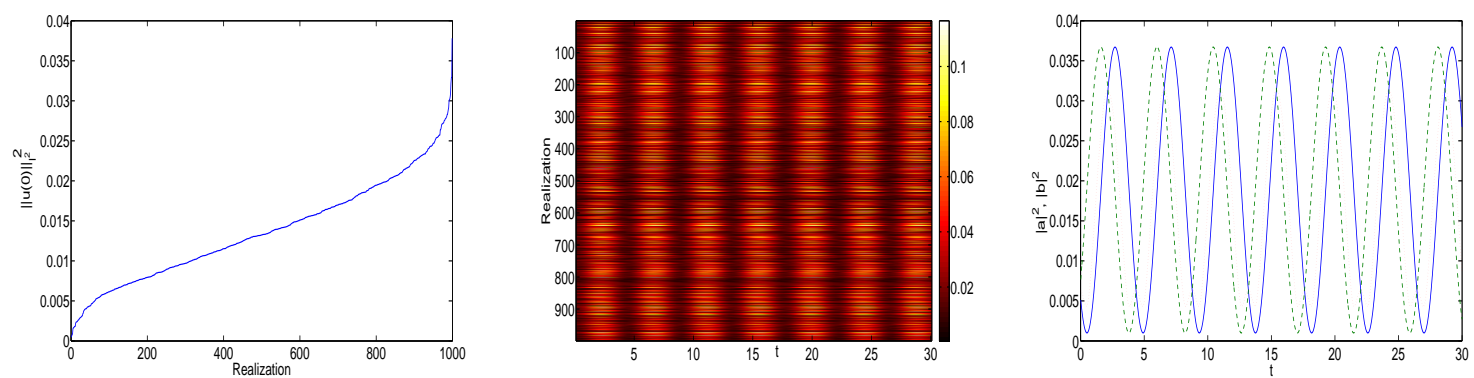

Figure 1: The left panel shows the (sorted in increasing value) squared numerical $l^{2}$ norm for our 1000 realizations of uniformly distributed random initial data whose real and imaginary parts are drawn within the interval $[0,0.1]$. The evolution of the different initial data is shown as a colormap of the total density $|a(t)|^{2}+|b(t)|^{2}$ versus time (in $[0,30]$ ) and the realization index in the middle panel. The bounded nature of the results attests to the absence of indefinite growth and the existence of oscillations. The right panel shows a typical example of bounded oscillations for $|a(t)|^{2}$ (solid blue line) and $|b(t)|^{2}$ (green dashed line).

\section{Numerical illustrations for dimers and quadrimers}

We now turn to a numerical illustration of the analytical results R1-R3. The case of the dimer is considered in Figures 1 and 2. For demonstration purposes, we choose $\gamma=0.7<1$, although we have verified the validity of the results also for other values of $\gamma$ in the interval $(0,1)$.

In Fig. 1, we explore the fate of sufficiently small initial data in connection with the statement R2. In particular, we sample both the real and the imaginary parts of the initial data from a uniform distribution in the interval $[0,0.1]$. As a result, for all 1000 realizations considered herein the squared initial $l^{2}$ norm is less than 0.04 , and hence this case corresponds to the choice $\delta=0.2$. We can see in the middle panel of the figure depicting the evolution of all 1000 realizations in time via a contour plot of $|a(t)|^{2}+|b(t)|^{2}$ that this quantity remains bounded (notice also the relevant colorbar). Moreover, it typically appears to feature oscillatory dynamics, a canonical example of which is featured on the right panel of the figure. Hence, in accordance with the statement R2, solutions for all sufficiently small initial data remain bounded for the monitored times.

In Fig. 2, we explore a setting where the initial data are sampled from an interval ten times as large, that is, the real and imaginary parts of $a$ and $b$ are drawn randomly from a uniform distribution in the interval $[0,1]$. This enables the monitoring of large initial data settings as is clearly illustrated in the figure (based on the corresponding initial norms). This, in turn, leads a large fraction of the initial data to grow exponentially over time, verifying the statement R3; these solutions are illustrated by a saturated white color in the contour plot of $\log \left(|a(t)|^{2}+|b(t)|^{2}\right)$ in the top right panel of Fig. 2, On the other hand, there are still among these 1000 realizations ones that preserve roughly the same (red) colormap throughout their evolution, indicating that they correspond to bounded solutions. An example of each of these two possibilities is illustrated in the bottom panels of Fig. 2. On the bottom left panel, a solution consonant with statement R3 (in that it is associated with exponential growth) and also with statement $\mathrm{R} 1$ (in that the growth happens with rate $2 \gamma$ shown for comparison by a 

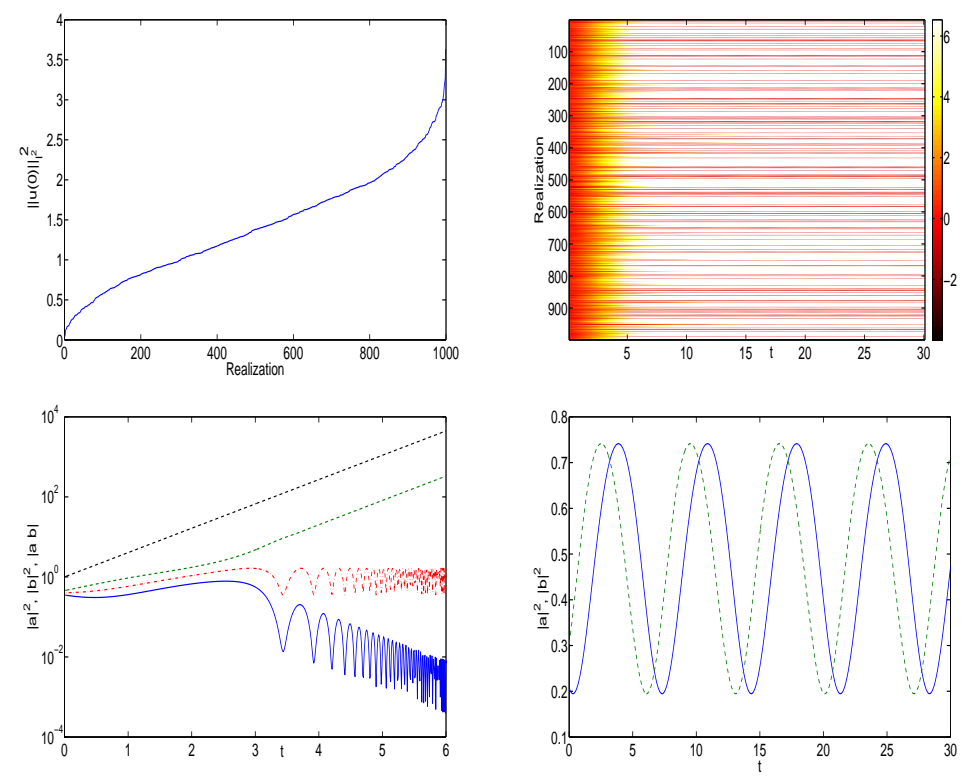

Figure 2: The same as in Figure 1 but the initial amplitudes of both real and imaginary parts are taken to be ten times larger (i.e., drawn randomly from a uniform distribution in $[0,1]$ ). The top left panel shows the (sorted) distribution of the initial square $l^{2}$ norms. The top right panel shows the time evolution of the different realizations. In this case the contour plot shows $\log \left(|a(t)|^{2}+|b(t)|^{2}\right)$ and the results are saturated for large norms; i.e., for the realizations resulting in the white regions, the evolution results in indefinite growth, while for the "red threads", the evolution stays bounded for all $t$. The bottom panels show a respective example of the two possible scenaria (in the same form as in Fig. 1). The only difference is that in the left panel for comparison a dashed black line is used to depict $e^{2 \gamma t}$ in the semilog plot, clearly indicating that the growth rate of $|b(t)|^{2}$ asymptotically follows the theoretical prediction. The bounded product $|a(t) b(t)|$ is shown also in the form of a (red) dash-dotted line.

black dash-dotted line) is illustrated. It is also worthwhile to note that for such solutions, the product $|a(t) b(t)|$ respects the bounded evolution, whereas $|a(t)|$ decreases exponentially with some oscillations. On the bottom right panel, an oscillatory and bounded solution is shown.

We now turn our attention to the case of quadrimers. The case of small initial data for the quadrimer is examined in Fig. 3. Once again, we have monitored the evolution of 1000 quadrimer realizations, with random initial data chosen from a uniform distribution with both real and imaginary parts in $[0,0.1]$. It can be clearly seen from the colorbar that all relevant runs maintain a norm which is bounded and follow what appears to be a nearly periodic evolution (see, in particular, a typical case example in the right panel of Fig. 3).

On the other hand, Figure 4 shows dynamics of the quadrimer starting with random initial data of size ten times larger than in Figure 3. In this case, similarly to Fig. 2. we have plotted the squared $l^{2}$ norm of the chain in a logarithmic contour plot, with the saturation (i.e., the yellow/faint color) indicating the indefinite growth of most configurations. On the other hand, in this case too, a number of solutions (the ones appearing as "red threads" in the left panel 

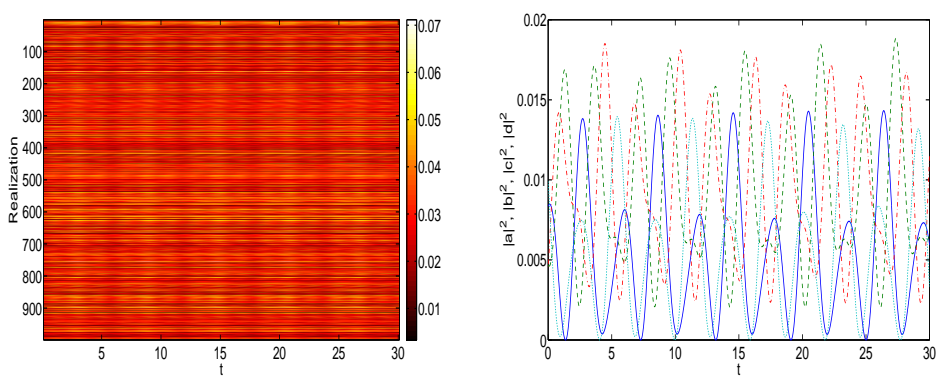

Figure 3: Same as Figure 1 but for the quadrimer with small initial data. For the contour plot evolution of the squared $l^{2}$ norm, it is clear that all orbits remain bounded (left panel). A typical example of the resulting bounded orbit is shown in the right panel with the blue solid and red dash-dotted lines denoting the gain sites, while the green dashed and cyan dotted lines correspond to the lossy ones.
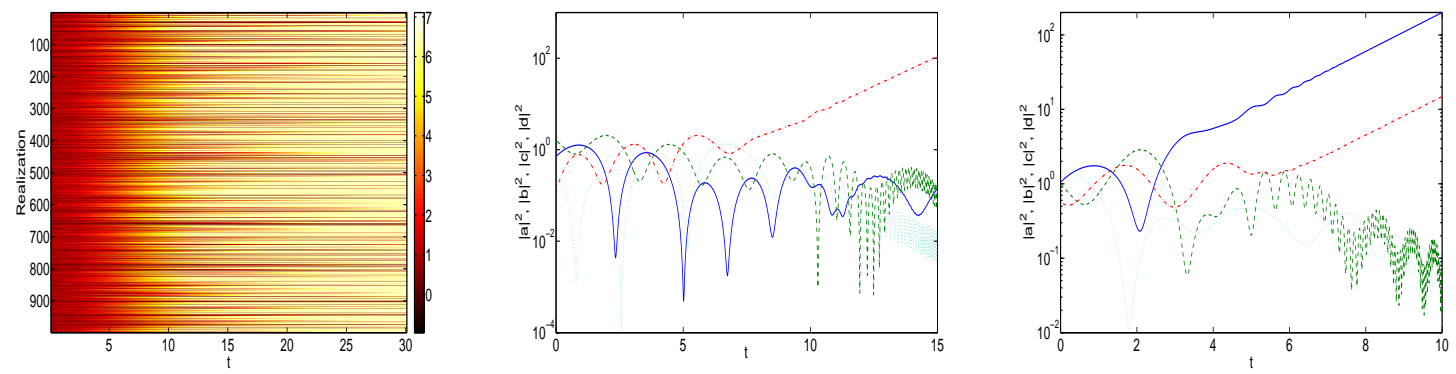

Figure 4: Same as in Figure 3 for the quadrimer with large initial data. From the saturation of the left panel's logarithmic scale, it is clear that most trajectories lead to indefinite growth. The middle and right panels illustrate the two principal scenaria through which this is happening i.e., one of the gain sites growing exponentially (middle) or both of the gain sites growing exponentially (right).

of Fig. 4) remain bounded. The two additional panels of Fig. 4. middle and right, display the two prototypical scenarios that we have observed as being realized when indefinite growth (according to the predicted rate of $e^{2 \gamma t}$ for the squared densities) arises for the quadrimer configurations. In the middle panel, only one of the two gain sites ultimately grows, while the other only results in bounded oscillations. Nevertheless, in a number of the relevant cases, like the one of the right panel, it is clear that both gain sites are ultimately led towards indefinite growth with their lossy counterparts both decaying in this case.

\section{Discussion}

In the present work, we have explored the dynamical features of the finite PT-dNLS chains. We have centered our exposition around three principal features of the models. 
Using an a priori estimate, based on the generalization of the $l^{2}$ norm (former for $\gamma=0$ ) conservation law, and the Gronwall lemma, we precluded finite time blowup for this system and offered an upper bound on its potential growth.

Secondarily, we were able to provide sharp bounds for the maximal possible growth of the amplitude at the sites with gain and the decay of the amplitudes at the sites with loss, making the interesting observation that the product of the two stays bounded. This analysis, in turn, permitted us to explore the fate of small data, leading to the conclusions that solutions starting with such data always remain bounded, as may be intuitively anticipated on the basis of the spectral stability of the zero equilibrium (in the regime of exact PT-symmetry).

Finally, perhaps the most counter-intuitive of our findings concerned the potential indefinite growth of solutions with large initial data even in the regime of exact PT phase. Not only were we able to offer sufficient conditions for such growth, but also we were able to identify its precise rate, revealing that the growth rate indicated by the priori bound is not merely an upper bound but a precise characterization of the density increase.

These results were initially presented in systematic detail in the case of the dimer; in the latter, the complete integrability revealed earlier in [16] can be used to make considerations far more precise, as indicated in Appendix A. However, we intentionally chose to present proofs of our statements that would generalize to more sites to indicate the generality of our considerations. All of these findings were also corroborated by systematic numerical computations, through a large number of realizations (with random initial data). All of these remained bounded for small initial data, as per statement R2, but for large initial data were led to indefinite growth both in the case of the dimer and in that of the quadrimer with the precise rate offered by statements R1 and R3.

It will certainly be relevant to extend the present considerations to a number of different directions. On the one hand, it would be interesting to complement considerations such as the ones presented herein with more precise estimates. In particular, offering sharper conditions for the "separatrices" between indefinite growth and bounded oscillations is a particularly interesting problem. On the other hand, the stable coherent structures of the finite chain would naturally be anticipated to be the centers around which the bounded motion is organized; however, aside from the integrable case of the dimer, this feature is not immediately transparent from our analysis and would be quite relevant to further explore. Finally, it is an interesting problem to consider higher dimensional settings, such as those in [10]. The latter settings may enable the formation of more complex phase patterns and their enhanced connectivity may modify the arguments devised herein.

\section{A Conservation quantities for a dimer}

We shall derive conserved quantities of the system of dimer equations (2) and use them to prove the three main results R1-R3. Note that the conserved quantities of this system were originally reported in [16] where they were introduced with the use of the so-called Stokes variables.

Separating the amplitudes and phases of the components $(a, b)$ of the system (2), we define new variables as follows:

$$
a(t)=\xi(t) e^{i \varphi(t)}, \quad b(t)=\eta(t) e^{i \varphi(t)+i \zeta(t)}
$$


Eliminating $\varphi$, we obtain an autonomous dynamical system for $(\xi, \eta, \zeta)$ :

$$
\left\{\begin{array}{l}
\dot{\xi}=-\gamma \xi+\eta \sin (\zeta), \\
\dot{\eta}=\gamma \eta-\xi \sin (\zeta), \\
\dot{\zeta}=\left(\eta^{2}-\xi^{2}\right)\left[\frac{\cos (\zeta)}{\xi \eta}-1\right],
\end{array}\right.
$$

where the dot corresponds to the time derivative in $t$.

Equilibrium states correspond to $\xi=\eta=a$ and $\sin (\zeta)=\gamma$ with two branches corresponding to $\cos (\zeta)= \pm \sqrt{1-\gamma^{2}}$ and an arbitrary positive parameter $a$. Linearization at the equilibrium states results in the characteristic equation with

$$
\lambda^{2}=-4 \cos (\zeta)\left(\cos (\zeta)-a^{2}\right)=\mp 4 \sqrt{1-\gamma^{2}}\left( \pm \sqrt{1-\gamma^{2}}-a^{2}\right)
$$

The upper branch is stable for $a^{2}<\sqrt{1-\gamma^{2}}$ and unstable for $a^{2}>\sqrt{1-\gamma^{2}}$. The lower branch is stable for all $a \in \mathbb{R}_{+}$.

Following [16], we find two conserved quantities of the third-order system (39), which enable us to find integral curves on the phase plane $(\xi, \eta)$. The first conserved quantity is

$$
E^{2}:=\xi^{2} \eta^{2}-2 \xi \eta \cos (\zeta)+1=(\xi \eta-\cos (\zeta))^{2}+\sin ^{2}(\zeta) \geq 0
$$

which is checked by direct differentiation. This conserved quantity can be used to eliminate variable $\zeta$.

The other conserved quantity follows from the balance equation

$$
\frac{d}{d t}\left[\xi^{2} \eta^{2}-2 E \sin \left(\frac{Q+\xi^{2}+\eta^{2}}{2 \gamma}\right)\right]=2\left(\eta^{2}-\xi^{2}\right)\left[\xi \eta \sin (\zeta)-E \cos \left(\frac{Q+\xi^{2}+\eta^{2}}{2 \gamma}\right)\right],
$$

where $Q$ is another arbitrary constant. Therefore,

$$
\left\{\begin{array}{l}
E \cos \left(\frac{Q+\xi^{2}+\eta^{2}}{2 \gamma}\right)=\xi \eta \sin (\zeta), \\
E \sin \left(\frac{Q+\xi^{2}+\eta^{2}}{2 \gamma}\right)=\xi \eta \cos (\zeta)-1,
\end{array}\right.
$$

the compatibility condition of which is equivalent to the conserved quantity (40).

The equilibrium states above correspond now to parametrization

$$
E^{2}=a^{4} \mp 2 a^{2} \sqrt{1-\gamma^{2}}+1, \quad E \cos \left(\frac{Q+2 a^{2}}{2 \gamma}\right)=\gamma a^{2},
$$

where parameter $a \in \mathbb{R}_{+}$is arbitrary and $(E, Q)$ are defined in terms of $a$. On the other hand, we can think about parameter $Q$ as arbitrarily fixed, then the system above fixes $a$ from the roots of a transcendental equation. This change in the point of view is important in obtaining integral curves on the phase plane $(\xi, \eta)$.

Proof of R1: If $(\xi, \eta)$ are initially positive and remain positive for the time span $[0, T]$, then the third equation of system (39) gives a bounded solution for $\zeta$ on $[0, T]$, whereas the first two equations of system (39) are bounded by linear functions in $(\xi, \eta)$. This allows us to construct an upper solution for $(\xi, \eta)$, which exists for all finite $t$ and grows exponentially in $t$. 
As a result, the time span $[0, T]$ is extended to $[0, \infty)$ provided that the solution $(\xi, \eta)$ remain positive for all $[0, \infty)$.

To justify the positivity of $(\xi, \eta)$, we use the conserved quantity (40). If $E^{2} \neq 1$, then $(\xi, \eta)$ cannot vanish due to the conservation of $E^{2}$ in (40) and hence the local solution is extended to all $t \in[0, \infty)$. The exceptional case $E^{2}=1$ has to be treated separately.

If $E^{2}=1$ and $(\xi, \eta)$ are initially positive, then the initial data and the local solution belong to the manifold in $\mathbb{R}^{3}$ :

$$
\xi \eta=2 \cos (\zeta) .
$$

Along the manifold (43), system (39) is rewritten in the equivalent form:

$$
\left\{\begin{array}{l}
\dot{\xi}=-\gamma \xi+\eta \sin (\zeta) \\
\dot{\eta}=\gamma \eta-\xi \sin (\zeta) \\
\dot{\zeta}=-\frac{1}{2}\left(\eta^{2}-\xi^{2}\right)
\end{array}\right.
$$

Again, $\zeta$ is defined for all $t$, for which a solution $(\xi, \eta)$ exists, and the upper solution for $(\xi, \eta)$ exists for all finite $t$ and grows exponentially in $t$. As a result, the local solution is extended to $[0, \infty)$, even if $\xi$ or $\eta$ or both change sign at a finite time instance $t_{0}>0$.

Proof of R3: Let $x=\xi^{2}+\eta^{2}$ and $y=\eta^{2}-\xi^{2}$ be new dynamical variables. Excluding $\sin (\zeta)$ by using the first equation in system (41), we transform the first two equations of system (39) to the equivalent form

$$
\left\{\begin{array}{l}
\dot{x}=2 \gamma y, \\
\dot{y}=2 \gamma x-4 E \cos \left(\frac{Q+x}{2 \gamma}\right) .
\end{array}\right.
$$

This system, where $(Q, E)$ are given, is cast to the second-order equation

$$
\ddot{x}=4 \gamma^{2} x-8 \gamma E \cos \left(\frac{Q+x}{2 \gamma}\right),
$$

with the first integral in the form

$$
I:=(\dot{x})^{2}-4 \gamma^{2} x^{2}+32 \gamma^{2} E \sin \left(\frac{Q+x}{2 \gamma}\right) .
$$

We note that $I=-16 \gamma^{2}\left(1+E^{2}\right)$ in connection to the conserved quantities (40) and (41).

Equilibrium states $\left(x_{0}, 0\right)$ are found from the transcendental equation

$$
\gamma x_{0}=2 E \cos \left(\frac{Q+x_{0}}{2 \gamma}\right)
$$

which agrees with the second equation in system (42) by the correspondence $x_{0}=2 a^{2}$. Linearization at the equilibrium states leads to the characteristic equation

$$
\lambda^{2}=4 \gamma^{2}+4 E \sin \left(\frac{Q+x_{0}}{2 \gamma}\right)
$$

which is stable (unstable) if $x_{0}$ is a minimum (maximum) point of the effective energy

$$
V(x):=-4 \gamma^{2} x^{2}+32 \gamma^{2} E \sin \left(\frac{Q+x}{2 \gamma}\right) .
$$


Note that $V(x) \sim-4 \gamma^{2} x^{2}$ for large $x$, no matter what $(Q, E)$ are. Therefore, all trajectories except for those trapped in local minima of $V(x)$ are unbounded in the variable $x=\xi^{2}+\eta^{2}$.

The unbounded solutions grow like $x(t) \sim e^{2 \gamma t}$ and $y(t) \sim e^{2 \gamma t}$ as $t \rightarrow \infty$, which implies that $\eta(t)$ grows exponentially in time $t$ like $\eta_{\infty} e^{\gamma t}$ as $t \rightarrow \infty$, where $\eta_{\infty}:=\lim _{t \rightarrow \infty} e^{-\gamma t} \eta(t)$. From conservation of $E^{2}$ in (40), we understand that $\xi(t)$ decays exponentially in time $t$ like $e^{-\gamma t}$ as $t \rightarrow \infty$. However, the actual behavior of $\xi(t)$ is complicated as is shown from the system (41) after eliminating $\zeta$ :

$$
\xi(t) \sim \frac{1}{\eta_{\infty}} \sqrt{1+E^{2}+2 E \sin \left(\frac{Q+\eta_{\infty}^{2} e^{2 \gamma t}}{2 \gamma}\right)} e^{-\gamma t} \text { as } \quad t \rightarrow \infty .
$$

In particular, the dynamics of $\xi(t)$ features rapid oscillations and exponential decay. Notice that this is in line with the numerical observations, as reported e.g. in the bottom left panel of Fig. 2.

Proof of R2: Assume that the initial data satisfies $\xi^{2}+\eta^{2} \leq \delta^{2}$ for a small parameter $\delta$. We shall first consider the dependence of conserved quantities $(E, Q)$ as functions of $(\xi, \eta)$. From (40), we have

$$
E=\sqrt{\xi^{2} \eta^{2}-2 \xi \eta \cos (\zeta)+1}=1-\xi \eta \cos (\zeta)+\mathcal{O}\left(\xi^{2}+\eta^{2}\right)^{2}
$$

In what follows, we write $E=1+\tilde{E}$, where $\tilde{E}=\mathcal{O}\left(\xi^{2}+\eta^{2}\right)=\mathcal{O}\left(\delta^{2}\right)$. From system (41) and expansion (50), we have

$$
Q=3 \pi \gamma+2 \gamma \xi \eta \sin (\zeta)-\xi^{2}-\eta^{2}+\mathcal{O}\left(\xi^{2}+\eta^{2}\right)^{2}
$$

Again, we write $Q=3 \pi \gamma+\tilde{Q}$, where $\tilde{Q}=\mathcal{O}\left(\xi^{2}+\eta^{2}\right)=\mathcal{O}\left(\delta^{2}\right)$. We emphasize again that $(\tilde{E}, \tilde{Q})$ are constants at the trajectory of the dynamical system (39) and the order of the expansion is indicated to measure its magnitude in terms of the magnitude of the initial data.

The integral curves of the dynamical system (45) are given by the first invariant (47), which is related to other conserved quantities by the relation $I=-16 \gamma^{2}\left(1+E^{2}\right)$. Substituting our decompositions for $(E, Q)$, we obtain the integral curves in the equivalent form

$$
y^{2}-x^{2}+16(1+\tilde{E}) \sin ^{2}\left(\frac{\tilde{Q}+x}{4 \gamma}\right)+4 \tilde{E}^{2}=0 .
$$

Expanding now the transcendental equation up to quadratic terms in the disk $0 \leq x \leq C \delta^{2}$ for some $C>0$ and hiding the residual terms of the $\mathcal{O}\left(\delta^{6}\right)$ magnitude, we obtain the quadratic form

$$
y^{2}+\frac{1}{\gamma^{2}}\left(\sqrt{1-\gamma^{2}} x+\frac{\tilde{Q}}{\sqrt{1-\gamma^{2}}}\right)^{2}=\frac{\tilde{Q}^{2}}{1-\gamma^{2}}-4 \tilde{E}^{2}+\mathcal{O}\left(\delta^{6}\right),
$$

where $\gamma \in(0,1)$ is assumed for linear stability of the zero equilibrium. Since the quadratic form is positive, all trajectories in the disk $0 \leq x \leq C \delta^{2}$ are closed curves. Therefore, all solutions are bounded for sufficiently small initial data $(\xi, \eta)$, implying nonlinear stability of the zero equilibrium in the dynamical system (39). 


\section{References}

[1] J. D'Ambroise, P. G. Kevrekidis, and S. Lepri, "Asymmetric wave propagation through nonlinear PT-symmetric oligomers", J. Phys. A Math. Theor. 45 (2012), 444012 (16 pages)

[2] I.V. Barashenkov, L. Baker, and N.V. Alexeeva, "PT-symmetry breaking in a necklace of coupled optical waveguides", Phys. Rev. A 87 (2013) 033819 (5 pages)

[3] S.V. Dmitriev, A.A. Sukhorukov, and Yu.S. Kivshar, "Binary parity-time-symmetric nonlinear lattices with balanced gain and loss", Opt. Lett. 35 (2010), 2976-2978.

[4] A. Guo, G. J. Salamo, D. Duchesne, R. Morandotti, M. Volatier-Ravat, V. Aimez, G. A. Siviloglou, and D. N. Christodoulides, "Observation of PT-Symmetry Breaking in Complex Optical Potentials", Phys. Rev. Lett. 103, 093902 (2009).

[5] P.G. Kevrekidis, D.E. Pelinovsky, and D.Y. Tyugin, "Nonlinear stationary states in PTsymmetric lattices", SIAM J. Appl. Dynam. Syst. (2013), accepted.

[6] D. Kip, private communication (2013).

[7] V.V. Konotop, D.E. Pelinovsky, and D.A. Zezyulin, "Discrete solitons in PT-symmetric lattices", EPL 100 (2012), 56006 (6 pages).

[8] M. Kulishov and B. Kress, "Free space diffraction on active gratings with balanced phase and gain/loss modulations", Optics Express 20 (2012), 29319-29328.

[9] K. Li and P.G. Kevrekidis, "PT-symmetric oligomers: analytical solutions, linear stability, and nonlinear dynamics", Phys. Rev. E 83 (2011), 066608 (7 pages).

[10] K. Li, P.G. Kevrekidis, B.A. Malomed, and U. Günther, "Nonlinear PT-symmetric plaquette", J. Phys. A Math. Theor. 45 (2012) 444021 (23 pages)

[11] A.E. Miroshnichenko, B.A. Malomed, and Yu.S. Kivshar, "Nonlinearly PT-symmetric systems: spontaneous symmetry breaking and transmission resonances", Phys. Rev. A 84 (2011), 012123 (4 pages).

[12] Z. H. Musslimani, K. G. Makris, R. El-Ganainy, and D. N. Christodoulides, Optical Solitons in PT Periodic Potentials, Phys. Rev. Lett. 100 (2008) 030402 (4 pages).

[13] S. Nixon, L. Ge, and J. Yang, "Stability analysis for solitons in PT-symmetric optical lattices", Phys. Rev. A 85 (2012), 023822 (10 pages).

[14] S. Nixon, Y. Zhu, and J. Yang, "Nonlinear dynamics of wave packets in parity-timesymmetric optical lattices near the phase transition point", Opt. Lett. 37 (2012), 48744876 .

[15] D.E. Pelinovsky, P.G. Kevrekidis, and D.J. Frantzeskakis, "PT-symmetric lattices with extended gain/loss are generically unstable", EPL 101 (2013), 11002 (6 pages).

[16] H. Ramezani, T. Kottos, R. El-Ganainy, and D.N. Christodoulides, "Unidirectional nonlinear PT-symmetric optical structures", Phys. Rev. A 82 (2010), 043803 (6 pages). 
[17] A. Ruschhaupt, F. Delgado, and J.G. Muga, "Physical realization of PT-symmetric potential scattering in a planar slab waveguide", J. Phys. A: Math. Gen. 38 (2005) L171-L176.

[18] C.E. Rüter, K.G. Makris, R. El-Ganainy, D.N. Christodoulides, M.Segev, and D. Kip, "Observation of parity-time symmetry in optics", Nature Physics 6 (2010) 192-195.

[19] S.V. Suchkov, B.A. Malomed, S.V. Dmitriev and Yu.S. Kivshar, "Solitons in a chain of parity-time-invariant dimers", Phys. Rev. E 84 (2011), 046609.

[20] A.A. Sukhorukov, S.V. Dmitriev, S.V. Suchkov, and Yu.S. Kivshar, "Nonlocality in PTsymmetric waveguide arrays with gain and loss" Opt. Lett. 37 (2012) 2148-2150.

[21] A.A. Sukhorukov, Z. Xu, and Yu.S. Kivshar, "Nonlinear suppression of time reversals in PT-symmetric optical couplers" Phys. Rev. A 82 (2010), 043818 (5 pages).

[22] D.A. Zezyulin and V.V. Konotop, "Nonlinear modes in finite-dimensional PT-symmetric systems" Phys. Rev. Lett. 108 (2012), 213906 (5 pages).

[23] M.C. Zheng, D.N. Christodoulides, R. Fleischmann and T. Kottos, "PT optical lattices and universality in beam dynamics", Phys. Rev. A 82 (2010), 010103(R). 